\title{
Duality for elliptic normal surface scrolls
}

\author{
Raquel Mallavibarrena ${ }^{1}$ and Ragni Piene ${ }^{2}$
}

\begin{abstract}
Let $C$ be a smooth, integral, complete curve, and consider a decomposable locally free sheaf on $C$. Given a realization of the associated projective vector bundle over $C$ as a ruled, nondevelopable variety, or scroll, in some projective space, we consider its higher order osculating spaces and dual varieties. We obtain results about the higher order dual varieties of such scrolls analogous to those obtained in the case of rational normal scrolls by Sacchiero and the second author [8]. These general results are then applied to scrolls defined by locally free rank 2 sheaves on an elliptic curve. If the sheaf is decomposable, we obtain directly a description of the dual varieties, in particular their dimension and degree. If the sheaf is indecomposable, it follows from a result of C. Segre [9] that the surface is the projection of a decomposable scroll, and we conclude with a comparison of the dual varieties of a scroll with those of its projection.
\end{abstract}

1. Introduction. If $X \subset \mathbf{P}^{N}$ is a projective variety, one defines $m$ th order dual varieties $X_{m}^{\vee} \subset\left(\mathbf{P}^{N}\right)^{\vee}$ as in [7] and [8]. The $m$ th order dual variety parametrizes hyperplanes having order of contact at least $m+1$ with $X$. Each such hyperplane contains an $m$ th order osculating space to $X$. In fact, at each smooth point $x \in X$ the various osculating spaces form a flag of linear subspaces, whose dimensions are independent of $x$ when $x$ is a general point [8, p.1042]. The dual variety corresponding to the largest of these linear spaces is called the strict dual variety and denoted by $X^{*}$.

Sacchiero and the second author [8] gave a complete description of the higher order dual varieties, in particular of the strict dual varieties, in the case that $X$ is a rational normal scroll. This description was obtained by considering a rational normal scroll of dimension $r$ as the variety swept out by $(r-1)$-dimensional linear spaces spanned by corresponding points on $r$ rational normal curves.

Our paper adds to the study of dual varieties of ruled varieties by considering more general cases. The description in [8] is given in terms of the behavior at a general point of $X$. We consider the varieties swept out by $(r-1)$-dimensional linear spaces spanned by corresponding points on $r$ linearly normal curves of genus $g$ and obtain analogous results. We call these varieties "decomposable normal scrolls" because they are defined, generalizing the rational case, by projectivizations of decomposable locally free sheaves on a curve $C$ of genus $g$.

${ }^{1}$ Supported in part by C.A.I.C.Y.T. PB86-0036 and in part by the Norwegian Research Council for Science and the Humanities.

${ }^{2}$ Supported in part by C.A.I.C.Y.T. PB86-0036.

1980 Mathematics subject classifications. Primary 14N10; Secondary 14N05, 14J26, 14 K07.

To appear in the Proceedings of the 1989 Zeuthen Symposium Comtemporary Mathematics, American Mathematical Society 
In Section 2 we make a local study of the homomorphisms defining the osculating spaces of decomposable scrolls using the definitions and techniques of [8]. We obtain a description of the "type" of the dual varieties $X_{m}^{\vee}$. In particular we determine their dimensions and degrees (Propositions 4, 5, 6). We also give necessary and sufficient conditions for reflexivity, the condition that the strict dual of the strict dual of the variety is equal to the variety itself (Proposition 7).

In Section 3 we study the case that $C$ is elliptic - this being the simplest case where indecomposable sheaves appear. We give necessary and sufficient conditions for the projectivization of a rank 2 locally free sheaf $\mathcal{E}$ over an elliptic curve $C$ to be embedded as a linearly normal scroll (Proposition 9). Here, we use Atiyah's classification [1] in terms of an invariant $e$ of the associated projective bundle. Moreover, following ideas of C. Segre [9], we realize every indecomposable normal elliptic scroll as the projection of a decomposable one from a point on it (Proposition 10).

In Section 4 we deduce our main duality results, the following two theorems.

TheOREM 1. If $X \subset \mathbf{P}^{2 d-e-1}$ is a decomposable normal elliptic scroll of dimension 2 , with invariant $e$ and degree $2 d-e$, where $d \geq e+3$, then

(i) if $e=0$, then $X^{*}=X_{d-1}^{\bigvee}$ is of dimension 2 and degree $2 d(d-1)$;

(ii) if $e=1$, then $X^{*}=X_{d-2}^{v}$ is of dimension 3 and degree $2 d^{2}-5 d+2$;

(iii) if $e \geq 2$, then $X^{*}=X_{d-2}^{v}$ is of dimension 1 and degree $d(d-1)$.

Theorem 2. If $X \subset \mathbf{P}^{2 d-e-1}$ is an indecomposable normal elliptic scroll of dimension 2, with invariant $e$ and degree $2 d-e$, where $d \geq e+3$, then

(i) if $e=-1$, then $X^{*}=X_{d-1}^{\mathrm{V}}$ is of dimension 3 and degree $2 d^{2}-3$;

(ii) if $e=0$, then $X^{*}=X_{d-1}^{v}$ is of dimension 2 and degree $2 d^{2}-d-2$.

To prove Theorem 2 we view the scroll as a (special) projection of a decomposable scroll (Proposition 10). We then apply a result relating the dual varieties of a scroll to those of its projection (Lemma 12).

Acknowledgments. We would like to thank Steven Kleiman and Gianni Sacchiero for their helpful remarks concerning the first version of this paper.

2. The dual varieties of decomposable scrolls. Fix an algebraically closed ground field of characteristic 0 (or of characteristic $p$, where $p$ is strictly greater than the integer $d_{r}$ below), and let $C$ be a smooth, integral, complete curve of genus $g$. For $i=1, \ldots, r$ let $\mathcal{M}_{i}$ be invertible sheaves on $C$ and assume that each $\mathcal{M}_{i}$ is generated by a subspace $V_{i}$ of $H^{0}\left(C, \mathcal{M}_{i}\right)$ so that we have surjections

$$
\left(V_{i}\right)_{C} \rightarrow \mathcal{M}_{i}
$$

Set $d_{i}:=\operatorname{dim} V_{i}-1$. Say $d_{1} \leq d_{2} \leq . . \leq d_{r}$. Let

$$
f_{i}: C \rightarrow \mathbf{P}\left(V_{i}\right)
$$

denote the corresponding morphisms, and set $C_{i}:=f_{i}(C)$.

Set $V:=\bigoplus_{i=1}^{r} V_{i}$ and $N:=\operatorname{dim} V=\sum_{i=1}^{r} d_{i}+r-1$. Set $\mathcal{M}:=\bigoplus_{i=1}^{r} \mathcal{M}_{i}$. The canonical surjection

$$
V_{C} \rightarrow \mathcal{M}
$$


induces a morphism

$$
f: \mathbf{P}(\mathcal{M}) \rightarrow \mathbf{P}(V)
$$

Set $X:=f(\mathbf{P}(\mathcal{M}))$.

By construction, $C_{i}$ is contained in $X$ for $i=1, \ldots, r$. Assume from now on that the morphisms $f_{i}$ are birational onto their images, then $f$ is also birational onto its image. Indeed, let $\pi: \mathbf{P}(\mathcal{M}) \rightarrow C$ denote the projection. Then for each $P \in C, f\left(\pi^{-1}(P)\right)$ is an $(r-1)$-dimensional linear space, or an $(r-1)$-plane, spanned by the points $f_{i}(P), i=1, \ldots, r$. Since the morphisms $f_{i}$ are generically injective, almost all these $(r-1)$-planes are distinct - in fact disjoint. Therefore $f$ is generically injective. Hence, in order to show that $f$ is birational onto its image, it suffices to show that it is generically unramified. This is equivalent to showing that the homomorphism $a^{1}$ defined below is generically surjective, and this holds because the maps $f_{i}$ are generically unramified by assumption.

Thus $X$ is a (possibly singular) variety ruled by $(r-1)$-planes - the generators of $X-$ in $\mathbf{P}(V)$. Note that by construction $X$ is not contained in any hyperplane. We call $X$ a decomposable scroll and write $X=\left(C_{1}, \ldots, C_{r}\right)$. The degree of $X$ is equal to

$$
c_{1}\left(\mathcal{O}_{\mathbf{P}(V)}(1)_{\mid X}\right)^{r}=c_{1}\left(\mathcal{O}_{\mathbf{P}(\mathcal{M})}(1)\right)^{r}=c_{1}(\mathcal{M})=\sum_{i=1}^{r} \operatorname{deg} \mathcal{M}_{i}
$$

The subspace $V$ of $H^{0}\left(\mathbf{P}(\mathcal{M}), \mathcal{O}_{\mathbf{P}(\mathcal{M})}(1)\right)$ defines a linear system on $\mathbf{P}(\mathcal{M})$. We call $X$ a decomposable normal scroll, if this linear system is complete.

LEMMA 3. If the linear system on $\mathbf{P}(\mathcal{M})$ corresponding to $V$ is complete, a sufficient condition for $f$ to be an embedding is that $\operatorname{deg} \mathcal{M}_{i} \geq 4 g+1$, for $i=1, \ldots, r$.

Proof. We adapt the argument for rational normal scrolls [8, Lemma 1, p. 1045]: It suffices to show that $\mathcal{O}_{\mathbf{P}(\mathcal{M})}(1)$ is very ample. Let $\mathcal{L}$ be an invertible sheaf on $C$ which is very ample and which is such that $\mathcal{M} \otimes \mathcal{L}^{-1}$ is generated by its global sections. Note that these assumptions hold if the degree of $\mathcal{L}$ is at least $2 g+1$ and $\operatorname{deg} \mathcal{M}_{i} \geq \operatorname{deg} \mathcal{L}+2 g$ for $i=1, \ldots, r$. Set $\mathcal{N}_{i}:=\mathcal{M}_{i} \otimes \mathcal{L}^{-1}$, set $W:=H^{0}\left(C, \oplus \mathcal{N}_{i}\right)$, and consider the sequence of embeddings

$$
\mathbf{P}\left(\oplus \mathcal{N}_{i}\right) \rightarrow C \times \mathbf{P}(W) \rightarrow \mathbf{P}\left(H^{0}(\mathcal{L})\right) \times \mathbf{P}(W) \rightarrow \mathbf{P}\left(H^{0}(\mathcal{L}) \otimes W\right),
$$

where the last map is the Segre embedding. Since the composition is an embedding, $\mathcal{L} \otimes \mathcal{O}_{\mathbf{P}\left(\mathcal{M} \otimes \mathcal{L}^{-1}\right)}(1)$ is very ample. But this invertible sheaf is isomorphic to $\mathcal{O}_{\mathbf{P}(\mathcal{M})}(1)$.

As with rational normal scrolls, we study the $m$ th order dual varieties of decomposable scrolls by studying the homomorphisms

$$
a^{m}: V_{X} \rightarrow \mathcal{P}_{X}^{m}(1)
$$

where $\mathcal{P}_{X}^{m}(1)$ denotes the sheaf of principal parts of order $m$ of the invertible sheaf $\mathcal{O}_{X}(1):=f^{*} \mathcal{O}_{\mathbf{P}(V)}($ see $[6,6$, p. 492] or $[4$, pp. 342-346]). The $m$ th order 
osculating space to $X$ at a point $x$ is defined by the image of the map $a^{m}(x)$, i.e., as $\mathbf{P}\left(\operatorname{Im} a^{m}(x)\right) \subset \mathbf{P}(V)$.

At a point $P \in C$, let $t$ denote a local (analytic) parameter for $C$ so that $f_{i}(P)=\left(x_{0}(0), \ldots, x_{d_{i}}(0)\right)$ for $i=1, \ldots, r$. Then for each $f_{i}: C \rightarrow \mathbf{P}\left(V_{i}\right)$ the homomorphism

$$
a_{f_{i}}^{m}:\left(V_{i}\right)_{C} \rightarrow \mathcal{P}_{C}^{m}\left(\mathcal{M}_{i}\right)
$$

is given locally around the point $P \in C$ by the matrix

$$
M_{d_{i}}^{m}=\left(\begin{array}{cccc}
x_{0}(t) & x_{1}(t) & \ldots & x_{d_{i}}(t) \\
x_{0}^{\prime}(t) & x_{1}^{\prime}(t) & \ldots & x_{d_{i}}^{\prime}(t) \\
\vdots & \vdots & \ddots & \vdots \\
x_{0}^{(m)}(t) & x_{1}^{(m)}(t) & \ldots & x_{d_{i}}^{(m)}(t)
\end{array}\right)
$$

The linear space spanned by the row vectors of this matrix for $t=0$ defines the $m$ th order osculating space to $C_{i}$ at the point $f_{i}(P)$.

We may choose coordinates on $\mathbf{P}\left(V_{i}\right)$ such that the orders of vanishing at $t=0$ of the functions $x_{i}(t)$ are strictly increasing. If $P$ is not a point of hyperosculation of $C$ with respect to the map $f_{i}$, and if $m \leq d_{i}$, then

$$
M_{d_{i}}^{m}(0)=\left(\begin{array}{cccccc}
\alpha_{0} & 0 & \ldots & 0 & \ldots & 0 \\
* & \alpha_{1} & \ldots & 0 & \ldots & 0 \\
\vdots & \vdots & \ddots & \vdots & & \vdots \\
* & * & \ldots & \alpha_{m} & \ldots & 0
\end{array}\right)
$$

where the $\alpha_{i}$ 's are non-zero constants [5, (9), p. 57]. Note that if $m>d_{i}$, then any $m$ th order osculating space to $C_{i}$ is the whole space $\mathbf{P}\left(V_{i}\right)$.

Suppose now that $P=P(0) \in C$ is not hyperosculating for any of the maps $f_{i}$, and let $y$ be a point on the generator $X_{P}$ corresponding to $P$. Let $\lambda_{1}, \ldots, \lambda_{r}$ denote homogeneous coordinates obtained by trivializing the sheaf $\mathcal{M}$ around $P$. Then $X$ has a local parametrization $y\left(t ; \lambda_{1}, \ldots, \lambda_{r}\right)$ at $y$. Let $\bar{M}_{d_{i}}^{m-1}$ denote $M_{d_{i}}^{m-1}$ if $m-1 \leq d_{i}$ and $M_{d_{i}}^{d_{i}}$ otherwise. As in the case of rational normal scrolls, the matrix corresponding to the map $a^{m}$ at a point $y$ where $\lambda_{r}=1$, is row equivalent to

$$
\left(\begin{array}{c}
A_{m} \\
0
\end{array}\right)
$$

where $A_{m}$ is the matrix

$$
A_{m}=\left(\begin{array}{ccccc}
\bar{M}_{d_{1}}^{m-1} & 0 & \ldots & 0 & 0 \\
0 & \bar{M}_{d_{2}}^{m-1} & \ldots & 0 & 0 \\
\vdots & \vdots & \ddots & \vdots & \vdots \\
0 & 0 & \ldots & \bar{M}_{d_{r-1}}^{m-1} & 0 \\
\lambda_{1} M_{d_{1}}^{m} & . & \ldots & \lambda_{r-1} M_{d_{r-1}}^{m} & M_{d_{r}}^{m}
\end{array}\right) .
$$

We see that if $m \leq d_{1}$, then rk $a^{m}(x(t ; \lambda))=r m+1$, and that if $m \geq d_{r}+1$, then $\operatorname{rk} a^{m}(x(t ; \lambda))=N+1$. By setting $\lambda_{1}=\ldots=\lambda_{r-1}=0$, we see that the 
$m$ th order osculating space to $X$ at the point $y_{r}(t)=f_{r}(P(t))=C_{r} \cap X_{P(t)}$ is equal to the space spanned by the $(m-1)$ th order osculating spaces to $C_{i}$ at $y_{i}(t):=C_{i} \cap X_{P(t)}$, for $i=1, \ldots, r-1$, and the $m$ th order osculating space at $y_{r}(t)$, and similarly for the points $y_{i}(t)$ for $i \leq r-1$.

Recall some definitions from [8]. For each $m$, we let $s(m)$ denote the generic rank of the map $a^{m}$. Define the $m$ th order dual variety $X_{m}^{\vee}$ of $X$ as the closure in $\mathbf{P}\left(V^{\vee}\right)$ of the set of hyperplanes containing an $s(m)$-dimensional $m$ th order osculating space to $X$. We set

$$
\bar{s}:=\max \{s(m) \mid s(m)<N\}
$$

and put $\bar{m}=\min \{m \mid s(m)=\bar{s}\}$. The strict dual variety $X^{*}$ of $X$ is defined by $X^{*}=X \frac{\mathrm{V}}{m}$.

For each integer $m$ such that $1 \leq m \leq \bar{m}$, let $i(m)$ denote the integer such that $0 \leq i(m) \leq r-1$ and $d_{i(m)}+1 \leq m \leq d_{i(m)+1}$ where $d_{0}:=0$.

Given subvarieties $Y_{1}, \ldots, Y_{u}$ of some projective space, we let $\left[Y_{1}, \ldots, Y_{u}\right]$ denote the smallest linear subspace containing these varieties. For each ordered subset $\left(i_{1}, \ldots i_{s}\right)$ of $(1, \ldots, r)$, we let $X_{i_{1}, \ldots i_{s}}=\left(C_{i_{1}}, \ldots, C_{i_{s}}\right)$ denote the corresponding ruled variety in $\left[C_{i_{1}}, \ldots, C_{i_{s}}\right]=\mathbf{P}\left(V_{i_{1}} \oplus \ldots \oplus V_{i_{s}}\right)$.

Let $S^{1}\left(C_{r}\right)$ denote the tangent developable of the curve $C_{r}$. Let

$$
\left(C_{1}, \ldots, C_{r-1}, S^{1}\left(C_{r}\right)\right)
$$

denote the variety in $\mathbf{P}(V)$ swept out by the $r$-dimensional linear spaces spanned by the points $f_{1}(P), \ldots, f_{r-1}(P)$ and the tangent line to $C_{r}$ at $f_{r}(P)$ for $P \in C$.

The strict dual curve $C_{j}^{*} \subset \mathbf{P}\left(V_{j}^{\mathrm{V}}\right)$ of $C_{j} \subset \mathbf{P}\left(V_{j}\right)$ is equal to the curve

$$
\left(C_{j}\right)_{d_{j-1}}^{\vee} \cap\left[C_{1}, \ldots, \hat{C}_{j}, \ldots, C_{r}\right]^{\vee}
$$

under the natural identification $\mathbf{P}\left(V_{j}^{\vee}\right)=\left[C_{1}, \ldots, \hat{C}_{j}, \ldots, C_{r}\right]^{\vee}$.

The following proposition can be seen from the local description of the maps $a^{m}$; the proof is the same as for rational normal scrolls [8, Prop. 1, p. 1057].

Proposition 4. If $i(m) \leq r-2$, then

$$
\operatorname{dim} X_{m}^{\vee}=N+1-r m+\sum_{j=1}^{i(m)}\left(m-1-d_{j}\right) .
$$

If $i(m)=r-1$, then

$$
\operatorname{dim} X_{m}^{\vee}=N-r m+\sum_{j=1}^{r-1}\left(m-1-d_{j}\right)=d_{r}-m
$$

Moreover, if $i(m) \geq 1$, we have

$$
X_{m}^{\vee}=\left(X_{i(m)+1, \ldots, r}\right)_{m}^{\vee} \cap\left[X_{1, \ldots, i(m)}\right]^{\vee} .
$$


Proposition 5. The strict dual variety $X^{*}$ of $X$ has the following description, depending on the relation between $d_{r}$ and $d_{r-1}$.

a) Suppose $d_{r}=d_{r-1}$. Let $j$ be the integer such that $d_{j}<d_{j+1}=\ldots=d_{r-1}=d_{r}$. Then $\bar{m}=d_{r}$ and $i(\bar{m})=j$. Moreover,

$$
X^{*}=\left(C_{j+1}^{*}, \ldots, C_{r}^{*}\right) \subset\left[X_{1}, \ldots, X_{j}\right]^{\vee} .
$$

b) Suppose $d_{r}=d_{r-1}+1$. Let $j$ be the integer such that $d_{j}<d_{j+1}=\ldots=d_{r-1}$. Then $\bar{m}=d_{r-1}$ and $i(\bar{m})=j$. Moreover,

$$
X^{*}=\left(C_{j+1}^{*}, \ldots, C_{r-1}^{*}, S^{1}\left(C_{r}^{*}\right)\right) \subset\left[X_{1}, \ldots, X_{j}\right]^{\vee} .
$$

c) Suppose $d_{r} \geq d_{r-1}+2$. Then $\bar{m}=d_{r}-1$ and

$$
X^{*}=C_{r}^{*} \subset\left[X_{1, \ldots, r-1}\right]^{\vee} \text {. }
$$

Proof. The proof is similar to that of [8, Prop. 2, p. 1057].

Proposition 6. Let $\left(C_{i}\right)_{m-1}^{\vee} \subset \mathbf{P}\left(V_{i}^{\vee}\right)$ denote the $(m-1)$ th dual variety of the curve $C_{i} \subset \mathbf{P}\left(V_{i}\right)$. Then

$$
\operatorname{deg} X_{m}^{\vee}=\sum_{i=1}^{r} \operatorname{deg}\left(C_{i}\right)_{m-1}^{\vee} .
$$

Proof. Let $j$ be the largest integer such that $m>d_{j}$. For each integer $i>j$, let $L_{i} \subset \mathbf{P}\left(V_{i}\right) \subset \mathbf{P}(V)$ be a linear subspace of dimension $d_{i}-m-1$ in general position with respect to $C_{i}$. Set $L:=\left[L_{j+1}, \ldots, L_{r}\right]$. Then $L$ is a linear subspace of $\mathbf{P}(V) \cong \mathbf{P}^{N}$ of dimension $N-M-1$, where $M=\sum_{k=1}^{j} d_{k}+(r-j) m+r-1$, and $L$ is "general" with respect to $X$ in the sense that the projection $\bar{X} \subset \mathbf{P}^{M}$ of $X$ from $L$ satisfies $\bar{X}_{m}^{\vee}=X_{m}^{\vee} \cap L^{\vee}$. But $\bar{X}_{m}^{\vee}=\bar{X}^{*}$, and $\bar{X}$ falls into case a) of Proposition 5. Note that, by definition, $\left(C_{i}\right)_{m-1}^{\vee}=\emptyset$ if $m>d_{i}$.

Proposition 7. We have $X^{* *}=X$ if and only if $d_{1}=\ldots=d_{r-1}$ and either $d_{r}=d_{r-1}$ or $d_{r}=d_{r-1}+1$. In all other cases, $X^{* *}$ is a cone containing $X$.

PROoF. This assertion follows from Proposition 5, compare [8, Theorem, 1) and 3), p. 1043]. (Note that in case $b$ ) the study of $X^{* *}$ does not require the tangent developable to be decomposable.)

Remark 8. The preceding results imply that if $X$ is self-dual (i.e., isomorphic to its strict dual variety), then the $d_{i}$ 's are equal and all the curves $C_{i}$ are selfdual. In particular, if the maps $f_{i}$ are all linearly normal embeddings, $X$ is self-dual only if $C$ is rational.

3. Elliptic normal scrolls of dimension 2. Let $C$ be a smooth elliptic curve, and $\mathcal{E}$ a locally free sheaf of rank 2 on $C$. We study the dual varieties of the ruled surface $\mathbf{P}(\mathcal{E})$ with respect to various linearly normal projective embeddings using results of Atiyah [1], as explained in Hartshorne [3, Ch.V, 2]. In particular, we assume that $\mathcal{E}$ is normalized; that is, $H^{0}(C, \mathcal{E}) \neq 0$, but for all invertible sheaves $\mathcal{L}$ on $C$ with $\operatorname{deg} \mathcal{L}<0$, we have $H^{0}(C, \mathcal{E} \otimes \mathcal{L})=0$. Recall that $e:=-\operatorname{deg} \mathcal{E}$ is an invariant of $\mathbf{P}(\mathcal{E})$ [3, Ch. V, 2.8, p. 372]. If $\mathcal{E}$ is decomposable, $\mathcal{E}=\mathcal{O}_{\mathcal{C}} \oplus \mathcal{L}$ and $e=-\operatorname{deg} \mathcal{L} \geq 0$ [3, Ch. V, 2.11.2, 2.11.3, p. 374]. If $\mathcal{E}$ is indecomposable, there are only two possible values of $e$, namely, 0 and -1 , and $\mathcal{E}$ is uniquely determined [3, Ch. V, 2.15, p. 377]. 
Proposition 9. Let $\mathcal{M}$ be an invertible sheaf on $C$ of degree $d$. If $d \geq e+3$, then $\mathcal{O}_{\mathbf{P}(\mathcal{E} \otimes \mathcal{M})}(1)$ yields an embedding of $\mathbf{P}(\mathcal{E} \otimes \mathcal{M}) \cong \mathbf{P}(\mathcal{E})$ as a linearly normal scroll $X \subset \mathbf{P}^{2 d-e-1}$ of degree $2 d-e$. Moreover, any linearly normal embedding of $\mathbf{P}(\mathcal{E})$ as a scroll is of this type.

Proof. Let $\pi: \mathrm{P}(\mathcal{E}) \rightarrow C$ denote the natural projection, and let $C_{0}$ be a section of $\pi$ with $C_{0}^{2}=-e$. It is known that $\left(\pi^{*} \mathcal{M}\right)\left(C_{0}\right)=\pi^{*} \mathcal{M} \otimes \mathcal{O}_{\mathbf{P}(\mathcal{E})}(1)$ is very ample on $\mathbf{P}(\mathcal{E})$ if and only if $d \geq e+3$ [3, Ch. V, Ex. 2.12, p. 385]. Hence, if $d \geq e+3$, then $H^{0}\left(\mathbf{P}(\mathcal{E}), \pi^{*} \mathcal{M} \otimes \mathcal{O}_{\mathbf{P}(\mathcal{E})}(1)\right)$ defines a projective embedding such that the fibres of $\pi$ are lines. Let $X$ denote this linearly normal, embedded scroll. The degree of $X$ is equal to the degree of the sheaf $\mathcal{E} \otimes \mathcal{M}$ which is $2 d-e$. Using this and the Riemann-Roch theorem on the elliptic curve $C$, we find that $H^{0}\left(\mathbf{P}(\mathcal{E}), \pi^{*} \mathcal{M} \otimes \mathcal{O}_{\mathbf{P}(\mathcal{E})}(1)\right)$ is of dimension $2 d-e$ as desired.

As each embedding of $\mathbf{P}(\mathcal{E})$ as a scroll is given by $\left(\pi^{*} \mathcal{M}\right)\left(C_{0}\right)$ for some invertible sheaf $\mathcal{M}$ on $C$ [3, Ch. V, Prop. 2.9, p. 373], the proof is finished.

Following ideas of C. Segre [9], as explained by Edge [2, 278, p. 217], we shall now see that the study of the dual varieties of indecomposable elliptic scrolls reduces to the study of the dual varieties of decomposable ones and of their projections.

Proposition 10. Suppose $\mathcal{E}$ is indecomposable and $X \subset \mathbf{P}^{2 d-e-1}$ is an embedding of $\mathbf{P}(\mathcal{E})$ given by an invertible sheaf $\mathcal{M}$ on $C$ of degree $d \geq e+3$ as in Prop. 9. Then there exists a decomposable scroll $X^{\prime} \subset \mathbf{P}^{2 d-e}$ such that $X$ is the projection of $X^{\prime}$ from a point on $X^{\prime}$.

Proof. Note that the sections of $X$ of degree $d^{\prime}$ correspond to invertible quotients $\mathcal{E} \otimes \mathcal{M} \rightarrow \mathcal{M}^{\prime}$ with $\operatorname{deg} \mathcal{M}^{\prime}=d^{\prime}$, hence to invertible quotients $\mathcal{E} \rightarrow \mathcal{L}^{\prime}$ with $\operatorname{deg} \mathcal{L}^{\prime}=d^{\prime}-d$.

First assume that $e=-1$. Then we have an exact sequence

$$
0 \rightarrow \mathcal{O}_{C} \rightarrow \mathcal{E} \rightarrow \mathcal{O}_{C}(P) \rightarrow 0
$$

for some point $P$ on $C$. This sequence defines a section $C^{\prime}$ of $X$ of degree $d+1$. Since $\mathcal{E}$ is normalized, this section is of minimal degree. Indeed, suppose $\mathcal{E} \rightarrow \mathcal{L}^{\prime}$ is a 1 -quotient such that $\operatorname{deg} \mathcal{L}^{\prime} \leq 0$. If $\mathcal{K}$ denotes the kernel, we have $\operatorname{deg} \mathcal{K} \geq 1$, and we get

$$
0 \rightarrow \mathcal{O}_{C} \rightarrow \mathcal{E} \otimes \mathcal{K}^{-1} \rightarrow \mathcal{L}^{\prime} \otimes \mathcal{K}^{-1} \rightarrow 0
$$

Therefore $H^{0}\left(C, \mathcal{E} \otimes \mathcal{K}^{-1}\right) \neq 0$, which contradicts the fact that $\mathcal{E}$ was normalized.

Let $Q \in C, Q \neq P$. The Riemann-Roch theorem gives

$$
\operatorname{dim} H^{0}\left(C, \mathcal{E} \otimes \mathcal{O}_{C}(Q-P)\right)=1
$$

(Note that $h^{\mathbf{1}}\left(C, \mathcal{E} \otimes \mathcal{O}_{C}(Q-P)\right)=h^{0}\left(C, \mathcal{E}^{\vee} \otimes \mathcal{O}_{C}(P-Q)\right)=0$ since $\mathcal{E}$ has no 1 -quotients of degree $\leq 0$.) This section corresponds to an exact sequence

$$
0 \rightarrow \mathcal{O}_{C}(P-Q) \rightarrow \mathcal{E} \rightarrow \mathcal{L}_{Q} \rightarrow 0
$$


where $\mathcal{L}_{Q}$ has degree 1 . The sheaf $\mathcal{L}_{Q}$ must be locally free, since if not, it would have a locally free rank 1 quotient of nonpositive degree, but $\mathcal{E}$ is normalized. Thus we get a section $C_{Q}$ of $X$ of degree $d+1$.

We have

$$
\mathcal{L}_{Q}=\Lambda^{2} \mathcal{E} \otimes \mathcal{O}_{C}(Q-P)=\mathcal{O}_{C}(P) \otimes \mathcal{O}_{C}(Q-P)=\mathcal{O}_{C}(Q)
$$

Consider the exact sequence

$$
0 \rightarrow \mathcal{E} \rightarrow \mathcal{O}_{C}(P) \oplus \mathcal{O}_{C}(Q) \rightarrow \mathcal{R} \rightarrow 0
$$

where $\mathcal{R}$ is a sheaf of length 1 . The sheaf $\mathcal{R}$ is supported on the point $Q$; this claim can be seen from the following commutative diagram:

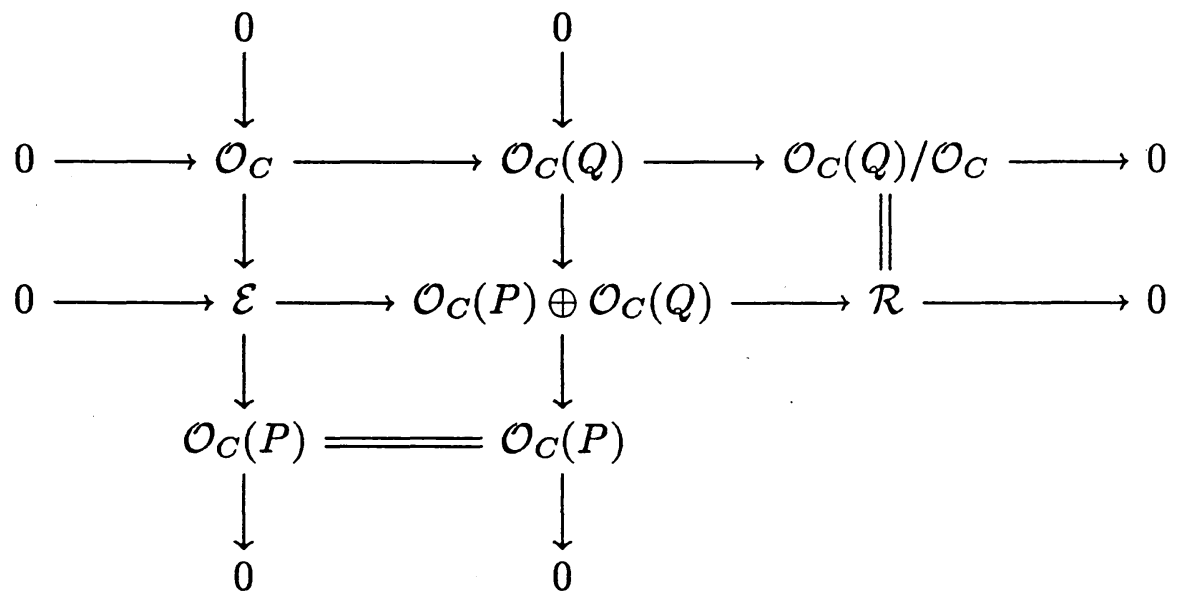

Define $X^{\prime}:=\mathbf{P}(\mathcal{M}(P) \oplus \mathcal{M}(Q)) \subset \mathbf{P}^{2 d+1}$. Then $X \subset \mathbf{P}^{2 d}$ is obtained from $X^{\prime}$ via projection from the point $R:=\mathbf{P}(\mathcal{R}) \in X^{\prime}$. Note that $X^{\prime}$ is decomposable, with invariant $e^{\prime}=0$, and that $X$ is an elementary transformation of $X^{\prime}$.

Assume next that $e=0$ and that $X \subset \mathbf{P}^{2 d-1}$ is given by an invertible sheaf $\mathcal{M}$ of degree $d \geq 3$. The exact sequence

$$
0 \rightarrow \mathcal{O}_{C} \rightarrow \mathcal{E} \rightarrow \mathcal{O}_{C} \rightarrow 0
$$

corresponds to a section $C_{0}$ of $X$ of degree $d$. Since $\mathcal{E}$ is normalized and indecomposable, this is a section of minimal degree, and it is unique.

Let $Q \in C$. By the Riemann-Roch theorem,

$$
\operatorname{dim} H^{0}\left(\mathcal{E} \otimes \mathcal{O}_{C}(Q)\right)=2
$$

Each section corresponds to an exact sequence

$$
0 \rightarrow \mathcal{O}_{C}(-Q) \rightarrow \mathcal{E} \rightarrow \mathcal{L}_{Q} \rightarrow 0
$$

where $\mathcal{L}_{Q}$ is a sheaf of degree 1 . If $\mathcal{L}_{Q}$ is not invertible, then it has an invertible quotient $\mathcal{L}$ of nonpositive degree. By the uniqueness of the minimal degree 
quotient of $\mathcal{E}$, the quotient $\mathcal{E} \rightarrow \mathcal{L}$ is equal to $\mathcal{E} \rightarrow \mathcal{O}_{C}$. Therefore, the surjection $\mathcal{L}_{Q} \rightarrow \mathcal{O}_{C}$ induces a homomorphism of exact sequences

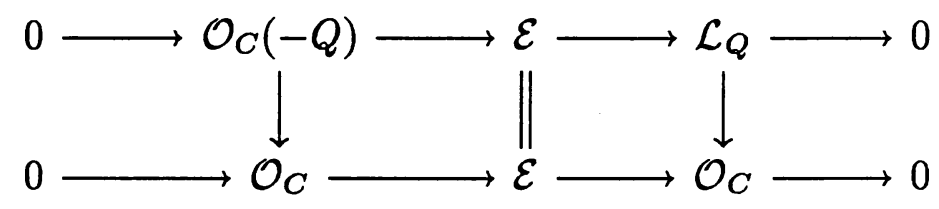

where the map $\mathcal{O}_{C}(-Q) \rightarrow \mathcal{O}_{C}$ is injective. Since there is only one such injection, there exists at most one section of $\mathcal{E} \otimes \mathcal{O}_{C}(Q)$ such that the corresponding sheaf $\mathcal{L}_{Q}$ is not invertible.

Thus we may take an invertible quotient

$$
\mathcal{E} \rightarrow \mathcal{L}_{Q}
$$

of degree 1 . We have

$$
\mathcal{L}_{Q}=\Lambda^{2} \mathcal{E} \otimes \mathcal{O}_{C}(Q)=\mathcal{O}_{C} \otimes \mathcal{O}_{C}(Q)=\mathcal{O}_{C}(Q) .
$$

This quotient corresponds to a section $C_{Q}$ of $X$ of degree $d+1$.

Consider the exact sequence

$$
0 \rightarrow \mathcal{E} \rightarrow \mathcal{O}_{C} \oplus \mathcal{O}_{C}(Q) \rightarrow \mathcal{R} \rightarrow 0
$$

Then

$\mathcal{R}$ is a sheaf of length 1 , and one sees, arguing as in the case $e=-1$ above, that $\mathcal{R}$ is supported on the point $Q$. Set $X^{\prime}:=\mathbf{P}(\mathcal{M} \oplus \mathcal{M}(Q)) \subset \mathbf{P}^{2 d}$. Then $X \subset \mathbf{P}^{2 d-1}$ is obtained via projection from the point $R:=\mathbf{P}(\mathcal{R}) \in X^{\prime}$. Note that $X$ is an elementary transformation of $X^{\prime}$, and $X^{\prime}$ is decomposable, with invariant $e^{\prime}=1$.

Remark 11. In both cases considered in the proof of Proposition $10(e=-1$ and $e=0)$, if $\pi^{\prime}: X^{\prime} \rightarrow C$ denotes the projection, then we have $\pi^{\prime}(R)=Q$.

4. Dual varieties of elliptic normal scrolls. In this section we prove Theorems 1 and 2 of the Introduction. Let $C$ be an elliptic curve and $\mathcal{E}$ a normalized rank 2 sheaf on $C$ with invariant $e$. Let $\mathcal{M}$ be an invertible sheaf of degree $d \geq e+3$ on $C$, and consider the corresponding linearly normal embedding of $\mathbf{P}(\mathcal{E})$ in $\mathbf{P}^{2 d-e-1}$ as a scroll of degree $2 d-e$. Denote this scroll by $X$.

To prove Theorem 1, assume $\mathcal{E}$ is decomposable. Consider first the "balanced" case, $e=0$. Then $X$ is of degree $2 d$ in $\mathbf{P}^{2 d-1}$. This scroll has two disjoint sections, $C_{1}$ and $C_{2}$, of degree $d$. Each $C_{i}$ is an elliptic normal curve in a linear subspace $\mathbf{P}^{d-1}$ of $\mathbf{P}^{2 d-1}$.

Recall that the strict dual curve of an elliptic normal curve of degree $d$ is a curve of degree $d(d-1)$ with $d^{2}$ ordinary cusps and no other singularities [6, Ex. 2, p. 480].

Applying Proposition 5 to $X$, with $r=2$ and $d_{i}=d-1$ for $i=1,2$, we get $\bar{m}=d-1$. Hence the strict dual variety of $X$ is the surface $X^{*}=X_{d-1}^{\vee}=$ $\left(C_{1}^{*}, C_{2}^{*}\right)$, and its degree is $2 d(d-1)$. 
Consider next the "semi-balanced" case, $e=1$. Then $X=\left(C_{1}, C_{2}\right) \subset \mathrm{P}^{2 d-2}$ is of degree $2 d-1$, with $\operatorname{deg} C_{1}=d_{1}+1=d-1$ and $\operatorname{deg} C_{2}=d_{2}+1=d$. Proposition 5 gives $X^{*}=X_{d-2}^{\vee}=\left(C_{1}^{*}, S^{1}\left(C_{2}^{*}\right)\right)$, so $X^{*}$ has dimension 3. Proposition 6 and $[6$, Ex. 2, p. 480] give

$$
\operatorname{deg} X^{*}=\sum_{i=1}^{2} \operatorname{deg}\left(C_{i}\right)_{d-3}^{\vee}=(d-1)(d-2)+d(d-2)=2 d^{2}-5 d+2
$$

If $e \geq 2$, then $\bar{m}=d-2$ and $X^{*}=X_{d-2}^{\vee}=C_{2}^{*}$. Hence $X^{*}$ is a curve of degree $d(d-1)$ with $d^{2}$ ordinary cusps and no other singularities [6, Ex. 2, p. 480]. Thus Theorem 1 is proved.

To prove Theorem 2, assume $\mathcal{E}$ is indecomposable. Then $e=-1$, or $e=0$. By Proposition 10 , if $\mathcal{M}$ is an invertible sheaf of degree $d \geq e+3$, then $X \subset \mathrm{P}^{2 d-e-1}$ is normal of degree $2 d-e$ and $X$ is the projection of a decomposable scroll $X^{\prime}$ in $\mathbf{P}^{2 d-e}$. By Theorem $1,\left(X^{\prime}\right)^{*}=\left(X^{\prime}\right)_{d-e-1}^{v}$. Finally, it follows from the local study of the maps $a^{m}$ for $X^{\prime}$ in $[8$, p. 1060] that almost all $m$ th osculating spaces of $X^{\prime}$ are of dimension $2 m$ for $m \leq d-e-1$.

If $e=-1$ and $m=d$, then the projection of almost every $d$ th osculating space of $X^{\prime}$ is the whole space $\mathbf{P}^{2 d}$ unless the center of projection $R$ belongs to all of them. But this is impossible because $\left(X^{\prime}\right)^{*}$ spans. $\left(\mathbf{P}^{2 d+1}\right)^{\vee}$ by Proposition 5 . Hence we conclude that $X^{*}=X_{d-1}^{\vee}$ holds.

If $e=0$ and $m=d-1$, then the projection of almost every $(d-1)$ th osculating space of $X^{\prime}$ is a $(2 d-2)$-dimensional space in $\mathbf{P}^{2 d-1}$, so again we get $X^{*}=X_{d-1}^{\vee}$.

To continue the proof of Theorem 2 we need the following variant of the "section and projection" theorem for duality [7, Prop. 2, p. 338].

LEMMA 12. Let $X^{\prime} \subset \mathbf{P}^{N+1}$ be a ruled surface not contained in a hyperplane, and let $X \subset \mathbf{P}^{N}$ be the projection of $X^{\prime}$ from a smooth point $R \in X^{\prime}$. Let $m<\left[\frac{N}{2}\right]$. Assume that almost all the $m$ th order osculating spaces to $X^{\prime}$ along the generator containing $R$ have dimension $2 m$ and are not constant along this generator. Let $H_{R}=\left(\mathbf{P}^{N}\right)^{\vee}$ denote the hyperplane of $\left(\mathbf{P}^{N+1}\right)^{\vee}$ dual to the point $R$, and let $X_{R}^{\prime}$ denote the generator containing the point $R$. Then

$$
\left(X^{\prime}\right)_{m}^{\vee} \cap H_{R}=X_{m}^{\vee} \cup\left(X_{R}^{\prime}\right)^{\vee}
$$

Moreover,

$$
\operatorname{deg} X_{m}^{\vee}=J \operatorname{deg}\left(X^{\prime}\right)_{m}^{\vee}-1
$$

Proof. The first equation is obvious for hyperplanes containing both $R$ and an $m$ th order osculating space to a point not on $X_{R}^{\prime}$. By assumption, almost all the $m$ th order osculating spaces to $X^{\prime}$ along $X_{R}^{\prime}$ have dimension $2 m$ and their union spans a $(2 m+1)$-dimensional linear subspace of $\mathbf{P}^{N+1}$. If $H^{\prime}$ is any hyperplane containing $X_{R}^{\prime}$, either $H^{\prime}$ contains this union, or $H^{\prime}$ intersects it in a linear space of dimension $2 m$. However, such a space must contain the $m$ th order osculating space to $X^{\prime}$ at some point of $X_{R}^{\prime}$. 
Example 13. Let $X$ be the quintic elliptic scroll in $\mathbf{P}^{4}$. Let $X^{\prime} \subset \mathbf{P}^{5}$ be a sextic elliptic scroll such that $X$ is the projection of $X^{\prime}$ from a point $R \in X^{\prime}$. Then $X^{*}=X^{\vee}$ has degree 5 , whereas $\left(X^{\prime}\right)^{\vee}$ has degree 6 . Let $H$ denote the hyperplane in $\mathbf{P}^{5}$ dual to $R$, and let $L$ denote the generator of $X^{\prime}$ containing $R$. Then $\left(X^{\prime}\right)^{\vee} \cap H=X^{\vee} \cup L^{\vee}$.

To apply Lemma 12 to prove Theorem 2, we need the following observation of C. Segre [9] (see also Edge [2, p. 218]).

LEMMA 14. Let $X \subset \mathbf{P}^{n-1}$ be a normal elliptic surface scroll of degree $n$. Then any section $D$ of $X$ of degree $c \leq n-1$ is an elliptic normal curve.

Proof. We must show that $D$ spans a $(c-1)$-dimensional linear subspace of $\mathbf{P}^{n-1}$. Set $\mathcal{O}_{X}(1):=\left.\mathcal{O}_{\mathbf{P}^{n-1}}(1)\right|_{X}$ and $\mathcal{O}_{D}(1):=\left.\mathcal{O}_{\mathbf{P}^{n-1}}(1)\right|_{D}$. Since $X$ is linearly normal, we have

$$
\operatorname{dim} H^{0}\left(X, \mathcal{O}_{X}(1)\right)=n
$$

Applying the Riemann-Roch theorem to the elliptic curve $D$, we get

$$
\operatorname{dim} H^{0}\left(D, \mathcal{O}_{D}(1)\right)=c
$$

Therefore it suffices to show that the restriction map

$$
H^{0}\left(X, \mathcal{O}_{X}(1)\right) \rightarrow H^{0}\left(D, \mathcal{O}_{D}(1)\right)
$$

is surjective, or equivalently that its kernel $H^{0}\left(X, \mathcal{O}_{X}(-D) \otimes \mathcal{O}_{X}(1)\right)$ has dimension $n-c$.

Suppose, in the notation of Proposition 9 , that $X=\mathbf{P}(\mathcal{E}) \cong \mathbf{P}(\mathcal{E} \otimes \mathcal{M})$. Let $\mathcal{E} \rightarrow \mathcal{L}^{\prime}$ denote the 1 -quotient corresponding to the section $D$, and define $\mathcal{K}$ by

$$
0 \rightarrow \mathcal{K} \rightarrow \mathcal{E} \otimes \mathcal{M} \rightarrow \mathcal{L}^{\prime} \otimes \mathcal{M} \rightarrow 0
$$

Then $\mathcal{K}$ is invertible and of degree $n-c$. Since by assumption $n-c \geq 1$, we have $H^{1}(C, \mathcal{K})=0$. Hence

$$
\operatorname{dim} H^{0}(C, \mathcal{K})=n-c
$$

However, by $[3$, Ch. V, 2.6, p. 371] we have

$$
\pi_{*}\left(\mathcal{O}_{X}(-D) \otimes \mathcal{O}_{X}(1)\right)=\mathcal{K}
$$

The proof is now complete.

We now finish the proof of Theorem 2. First consider the case $e=-1$. Then $X^{\prime}$ is decomposable and balanced, of type $\left(C^{\prime}, C_{Q}^{\prime}\right)$, where $C^{\prime}$ and $C_{Q}^{\prime}$ are elliptic normal curves of degree $d+1$. These curves have no points of hyperosculation of order $\leq d-1[6$, p. 480 and p. 483]. Now, it follows from the local description of the maps $a^{m}$ for $X^{\prime}$ in $[8$, p. 1060] that all the $m$ th osculating spaces are 
of dimension $2 m$ and non-constant along a generator for $m \leq d-1$. So the hypotheses of Lemma 12 are satisfied, and we obtain

$$
\operatorname{dim} X^{*}=\operatorname{dim}\left(X^{\prime}\right)_{d-1}^{\vee}-1=3 \text { and } \operatorname{deg} X^{*}=\operatorname{deg}\left(X^{\prime}\right)_{d-1}^{\vee}-1
$$

By Proposition 6 the degree of $\left(X^{\prime}\right)_{d-1}^{\vee}$ is equal to twice the degree of the $(d-2)$ th dual of an elliptic normal curve of degree $d+1$, hence to $2(d-1)(d+1)[6$, Thm. 3.2, p. 481]. Therefore,

$$
\operatorname{deg} X^{*}=2 d^{2}-3
$$

Next consider the case $e=0$. Let $C_{0} \subset X$ denote the minimal section; it is of degree $d$. Choose a point $Q$ on $C$ such that the corresponding point $Q_{0}$ on $C_{0}$ is not a point of hyperosculation. Choose a section $C_{Q}$ of degree $d+1$ as in the proof of Proposition 10. Then, by Propostion 10, $X$ is the projection of a decomposable and semi-balanced scroll $X^{\prime}$ from a point $R \in X^{\prime}$. Since $C_{0}$ lifts isomorphically to a section $C_{0}^{\prime}$ of $X^{\prime}$, the point $X_{R}^{\prime} \cap C_{0}^{\prime}$ is not a point of hyperosculation on $C_{0}^{\prime}$. The section $C_{Q}$ lifts to a section $C_{Q}^{\prime}$, which is an elliptic normal curve of degree $d+1$. Therefore $C_{Q}^{\prime}$ has no points of hyperosculation of order $\leq d-1$. Again, from the local description of osculating spaces in the decomposable case we know that the generator $X_{R}^{\prime}$ satisfies the hypotheses of Lemma 12 for $m \leq d-1$. Therefore

$$
\operatorname{dim} X^{*}=\operatorname{dim}\left(X^{\prime}\right)_{d-1}^{\vee}-1=2 .
$$

Since $\left(X^{\prime}\right)_{d-1}^{\vee}=\left(X^{\prime}\right)^{*}=\left(\left(C_{0}^{\prime}\right)^{*}, S^{1}\left(\left(C_{Q}^{\prime}\right)^{*}\right)\right)$, Proposition 6 implies

$$
\operatorname{deg} X^{*}=d(d-1)+(d-1)(d+1)-1=2 d^{2}-d-2 .
$$

The proof of Theorem 2 is now complete.

\section{REFERENCES}

[1] M. F. Atiyah, Vector bundles over an elliptic curve, Proc. London Math. Soc. 27 (1957), 414-452.

[2] W. L. Edge, "Ruled Surfaces," Cambridge University Press, 1931.

[3] R. Hartshorne, "Algebraic Geometry," Graduate Texts in Mathematics 52, SpringerVerlag, 1977.

[4] S. L. Kleiman, The enumerative theory of singularities, in "Real and complex singularities," P. Holm (ed.), Proc. Conf., Oslo 1976, Sijthoff \& Noordhoff, 1977, pp. 297-396.

[5] D. Laksov, Wronskians and Plücker formulas for linear systems on curves, Ann. Sci. École Norm. Sup. 17 (1984), 45-66.

[6] R. Piene, Numerical characters of a curve in projective $n$-space, in "Real and complex singularities," P. Holm (ed.), Proc. Conf., Oslo 1976, Sijthoff \& Noordhoff, 1977, pp. 475495.

[7] R. Piene, A note on higher order dual varieties, with an application to scrolls, in "Singularities," P. Orlik (ed.), Proc. Symposia Pure Math., Vol. 40, Amer. Math. Soc., 1983, pp. 335-342.

[8] R. Piene and G. Sacchiero, Duality for rational normal scrolls, Comm. Alg. 12 (1984), 1041-1066.

[9] C. Segre, Ricerche sulle rigate ellitiche di qualunque ordine, Atti della R. Acc. delle scienze di Torino 21 (1886), 868-891.

Dept. de Algebra, Facultad de Matematicas, Universidad Complutense, 28040 Madrid, Spain Matematisk Institutt, Universitetet i Oslo, Box 1053 Blindern, 0316 Oslo 3, Norway 